\title{
Influência ambiental sobre a incapacidade física: uma revisão sistemática da literatura
}

\author{
Environmental influence on physical disability: \\ a systematic review of the literature
}

Filipe Athayde ${ }^{1}$

Eliane Viana Mancuzo ${ }^{1}$

Ricardo de Amorim Corrêa ${ }^{1}$

${ }^{1}$ Programa de PósGraduação em Ciências Aplicadas à Saúde do Adulto, Faculdade de Medicina, Universidade Federal de Minas Gerais. Av. Pres. Antônio Carlos 6627, Pampulha. 31270-901 Belo Horizonte MG Brasil. ftsathayde@yahoo.com.br

\begin{abstract}
People with physical disabilities may, in interaction with facilitators and barriers, modify their participation in society. The environment, in the panorama of the International Classification of Functioning, Disability and Health (ICF), plays a relevant role in health and in the expression of functioning. Thus, the aim of this study was to investigate the influence of environmental factors on physical disability, based on the theoretical framework of the ICF. A systematic review of the literature was performed based on the recommendations of the following documents: Preferred Reporting Items for Systematic Reviews and $\mathrm{Me}$ ta-Analyses (PRISMA) and Strengthening the Reporting of Observational Studies in Epidemiology Statement (STROBE). The studies selected confirmed the theoretical precepts of the ICF. The interference of the physical and social environment, in general, might be attributed to different clinical conditions, especially in neurology, both in quantitative and qualitative studies. Methodological limitations were observed that may be obstacles to consolidating some guidelines, which should encourage further research on the subject with judicious methods, validated instruments and carefully selected samples.

Key words International Classification of Functioning, Disability and Health, Environment and public health, Disabled persons
\end{abstract}

Resumo Indivíduos com incapacidades físicas podem, em interação com facilitadores e barreiras, modificar a sua participação em sociedade. $O$ ambiente, no panorama da Classificação Internacional de Funcionalidade, Incapacidade e Saúde (CIF), apresenta-se com relevante papel na saúde e na expressão da funcionalidade. Dessa forma, o objetivo deste estudo foi investigar a influência de fatores ambientais sobre a incapacidade física, pautado no referencial teórico da CIF. Foi realizada uma revisão sistemática da literatura, norteada por recomendações dos documentos Preferred Reporting Items for Systematic Reviews and Meta-Analyses (PRISMA) e Strengthening the Reporting of Observational Studies in Epidemiology Statement (STROBE). Os estudos selecionados puderam reforçar os preceitos teóricos da CIF. O ambiente fisico e social, de modo geral, pôde ter sua interferência atribuída a diferentes condições clínicas, especialmente em neurologia, tanto em estudos quantitativos quanto qualitativos. Limitações metodológicas foram observadas e podem ser entraves à consolidação de alguns direcionamentos, o que deve incentivar novas pesquisas sobre o tema com métodos criteriosos, instrumentos validados e amostras cuidadosamente selecionadas.

Palavras-chave Classificação Internacional de Funcionalidade, Incapacidade e Saúde, Meio ambiente e saúde pública; Pessoas com deficiência 


\section{Introdução}

A incapacidade física pode ser compreendida de acordo com diferentes abordagens que visam esclarecer seus fatores causadores e consequências, com contribuições advindas principalmente dos modelos biomédico e social. De acordo com as conclusões da primeira convenção internacional da Organização das Nações Unidas sobre os direitos de pessoas com incapacidades, esses indivíduos apresentam deficiências físicas, mentais, intelectuais ou sensoriais, as quais podem, em interação com várias barreiras, reduzir sua participação em sociedade ${ }^{1}$.

Pautada no modelo biopsicossocial, a Classificação Internacional de Funcionalidade, Incapacidade e Saúde (CIF), adotada em 2001, representou uma evolução em relação ao entendimento dos diferentes contextos relacionados à deficiência física. Transcendendo a lógica biomédica baseada na etiologia das doenças e também a compreensão social dos processos de incapacidade, a noção de funcionalidade, segundo seus diversos componentes (condição de saúde, estrutura e função corporal, atividades e participação social), é influenciada pela interrelação entre fatores do contexto pessoal e ambiental. O conceito de incapacidade constitui denominação ampla neste processo e que, a partir da CIF, passou a ser considerada também em seu aspecto positivo, a funcionalidade, assim como o domínio ambien$\mathrm{tal}^{2,3}$. O ambiente, no panorama da CIF, apresenta-se com relevante papel na saúde e participação de indivíduos com incapacidades, podendo seus fatores ocorrer na forma de barreiras ou de facilitadores para expressão da funcionalidade ${ }^{4}$.

A qualidade de vida e a funcionalidade são influenciadas pela interação de características do indivíduo e de fatores ambientais ${ }^{5,6}$. Entretanto, há necessidade de reunir as informações disponíveis sobre o impacto do ambiente físico e social sobre a incapacidade, uma vez que a literatura atual envolve aspectos ambientais distintos e em populações variadas. Dessa forma, o objetivo deste estudo foi investigar, por meio de uma revisão de literatura, a influência de fatores ambientais sobre a incapacidade física, segundo o referencial teórico da CIF.

\section{Método}

Realizou-se uma revisão sistemática da literatura científica acerca da influência ambiental sobre a incapacidade física. Foram utilizadas as reco- mendações dos documentos Preferred Reporting Items for Systematic Reviews and Meta-Analyses (PRISMA) e Strengthening the Reporting of Observational Studies in Epidemiology Statement (STROBE), que orientam revisões sistemáticas e meta-análises na área da saúde, para nortear a avaliação das evidências científicas atualmente disponíveis.

Foram incluídos estudos originais que avaliaram primariamente o efeito do ambiente físico ou social sobre a mobilidade e a funcionalidade de indivíduos, crianças ou adultos, publicados entre 2010 e 2016. As bases de dados pesquisadas foram Lilacs, SciELO e PubMed. A estratégia de busca no PubMed foi a seguinte: (icf OR functioning) AND (environment OR environmental factors OR barriers OR facilitators) AND health, empregados os devidos filtros de acordo com critérios expostos. Nas plataformas latino-americanas, usou-se: ambiente AND (CIF OR funcionalidade $O R$ incapacidade); (barreiras $O R$ facilitadores) AND (CIF OR funcionalidade $O R$ incapacidade).

Os seguintes critérios de exclusão foram considerados: revisões de literatura; estudos metodológicos; core sets de acordo com itens da CIF; estudos de casos; pesquisas com desfechos direcionados à fala e linguagem.

\section{Resultados e discussão}

A partir de um registro inicial de 943 trabalhos encontrados nas buscas em bases de dados e um secundariamente localizado por referência em outro estudo, foram removidas as duplicidades e realizado o processo de seleção mediante os critérios previamente estabelecidos. Foram incluídos na revisão 18 artigos científicos originais, após leitura de resumos e textos completos, de acordo com as etapas apresentadas na Figura 1.

A literatura pesquisada foi composta de trabalhos desenvolvidos com populações de áreas clínicas diversificadas, com destaque para condições neurológicas em crianças e adultos. O Quadro 1 traz os estudos incluídos na revisão e seus respectivos perfis de participantes investigados.

Vários trabalhos avaliaram a contribuição do ambiente físico e social para a funcionalidade de crianças com deficiência. Gasparoto e Alpino ${ }^{7}$ pesquisaram as condições de acessibilidade e participação de um grupo de crianças com dificuldade de locomoção usuárias de equipamento de tecnologia assistiva no ambiente domiciliar. Por meio de check-list para mensurar a acessibilida- 


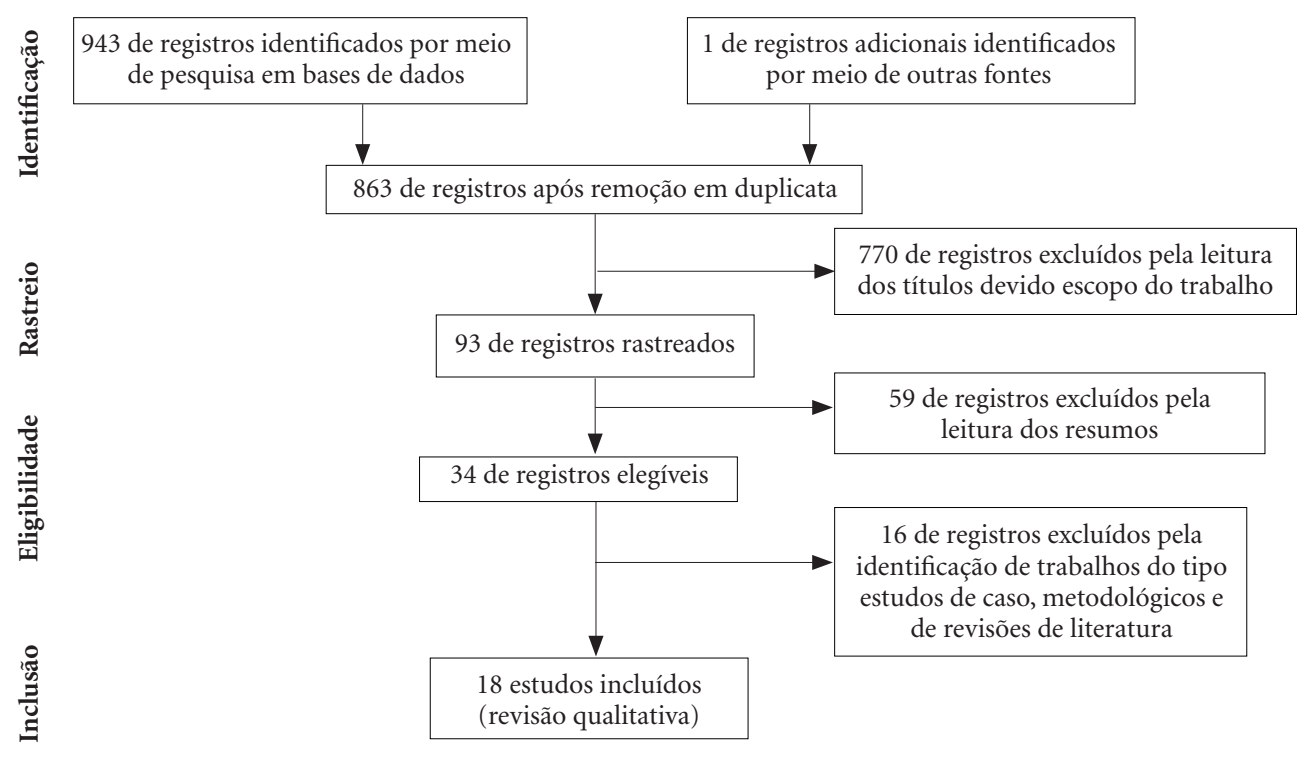

Figura 1. Fluxograma de fases da revisão sistemática.

Fonte: PRISMA Statement ${ }^{8}$, traduzido e adaptado.

Quadro 1. Estudos e áreas clínicas incluídos na revisão sistemática.

\begin{tabular}{|c|c|c|}
\hline Estudo & Área & Condições de Saúde \\
\hline Gasparoto e Alpino $^{7}$ & Neurologia Pediátrica & $\begin{array}{l}\text { Paralisia Cerebral, Malformação congênita e } \\
\text { Mielomeningocele }\end{array}$ \\
\hline Andrade et al. ${ }^{9}$ & Neurologia Pediátrica & Paralisia Cerebral e Acidente Vascular Encefálico \\
\hline Hammel et al. ${ }^{10}$ & Neurologia & $\begin{array}{l}\text { Lesão medular, Traumatismo Crânio-Encefálico e Acidente } \\
\text { Vascular Encefálico }\end{array}$ \\
\hline Kolehmainen et al. ${ }^{11}$ & Neurologia Pediátrica & Paralisia Cerebral e outros diagnósticos não especificados \\
\hline Rouquette et al. ${ }^{12}$ & Reumatologia & Osteoartrite de joelhosnão submetidos aartroplastia \\
\hline Zhang et al. ${ }^{13}$ & Neurologia & Acidente Vascular Encefálico \\
\hline Rantakokko et al. ${ }^{14}$ & Gerontologia & Idosos comunitários \\
\hline Bostan et al. ${ }^{15}$ & $\begin{array}{l}\text { Ortopedia e } \\
\text { Reumatologia }\end{array}$ & $\begin{array}{l}\text { Dor crônica difusa, Lombalgia, Osteoartrite, Osteoporose, } \\
\text { Artrite Reumatóide }\end{array}$ \\
\hline Chang et al. ${ }^{16}$ & Neurologia & Acidente Vascular Encefálico \\
\hline Rauch et al. ${ }^{17}$ & Neurologia & Lesão Medular \\
\hline den Ouden et al. ${ }^{18}$ & Gerontologia & $\begin{array}{l}\text { Condições de saúde variadas (cardiovasculares, neurológicas, } \\
\text { metabólicas, respiratórias, ortopédicas e neoplásicas) }\end{array}$ \\
\hline Sveen et al. ${ }^{19}$ & Neurologia & Traumatismo Crânio-Encefálico \\
\hline Virués-Ortega et al. ${ }^{20}$ & Gerontologia & Idosos comunitários \\
\hline Randström et al. ${ }^{21}$ & $\begin{array}{l}\text { Neurologia, Ortopedia } \\
\text { e Reumatologia }\end{array}$ & $\begin{array}{l}\text { Acidente Vascular Encefálico e condições ortopédicas } \\
\text { (predomínio de fraturas e artroplastias de quadril). }\end{array}$ \\
\hline Gallagher et al..$^{22}$ & Ortopedia & Amputações de membros (superior ou inferior). \\
\hline Rosenberg et al. ${ }^{23}$ & Neurologia Pediátrica & Incapacidades leves, sem condições clínicas especificadas \\
\hline Bouffioulx et al. ${ }^{24}$ & Neurologia & Acidente Vascular Encefálico \\
\hline Furtado et al. ${ }^{25}$ & Neurologia Pediátrica & Paralisia Cerebral \\
\hline
\end{tabular}

de nos domicílios, previamente testado mas não validado, com uma amostra pequena composta por apenas cinco crianças do sexo feminino (ida- de entre quatro e 12 anos), foi detectada carência de mobiliário e/ou recursos adaptados, apesar da frequente indicação; bem como predomínio 
de barreiras físicas tais como desníveis, aclives e pisos lisos nas rotas de acesso, inadequação da largura de portas de banheiros e redução do espaço de circulação nos cômodos do domicílio. $\mathrm{O}$ estudo de Andrade et al. ${ }^{9}$, ao avaliar o desempenho cognitivo, a funcionalidade e as percepções dos pais quanto aos facilitadores para reabilitação utilizando o questionário AFARNI (Avaliação dos Fatores Ambientais relacionados à Reabilitação Neurológica Infantil), com uma amostra de crianças com idade entre cinco e 18 anos, com Paralisia Cerebral (PC) ou Acidente Vascular Encefálico (AVE), pôde-se identificar que os pais de crianças com déficits cognitivos percebem de forma mais significativa a necessidade de apoio de profissionais e serviços especializados em relação àquelas com déficit motor. Kolehmainen et al. ${ }^{11}$ estudaram a relação do ambiente e a participação no brincar de 195 crianças com deficiências motoras (com PC, em sua maioria, e algumas sem diagnóstico firmado), de seis a oito anos, com locomoção independente assistida ou não por equipamentos. A participação no brincar, mensurada por questionário validado, apresentou correlação significativa com os seguintes fatores ambientais: ter amigos para brincar; família imediata (comportamento dos pais no brincar sem as crianças e comportamento de toda família no brincar). Por outro lado, Rosenberg et al. ${ }^{23}$ concluíram que os pais perceberam os fatores ambientais com pequeno impacto sobre a participação de seus filhos. Este estudo incluiu 79 crianças pré-escolares (média 5,20 $\pm 0,5$ anos) com incapacidades leves, sem especificar claramente seu modo de classificação. Os questionários, desenvolvidos e validados previamente pelos próprios autores, apontaram correlação negativa fraca entre a participação das crianças e barreiras físicas domésticas e para educação, além da ausência de associação significativa com barreiras da comunidade.

Outro trabalho envolvendo crianças, de Furtado et al..$^{25}$, investigou o efeito moderador dos fatores ambientais sobre a relação entre mobilidade e participação na escola de 102 crianças e adolescentes com PC, estudantes do ensino fundamental, com função motora grossa dos níveis I, II e III do Gross Motor Classification System. A mobilidade apresentou forte associação com participação escolar mensurada pelo School Function Assessment, entretanto não foi comprovada moderação do ambiente nesta relação. O pequeno impacto de fatores ambientais sobre a participação e a ausência de moderação ambiental foram atribuídos, pelos próprios autores, à insuficiente investigação pautada em aspectos negativos do ambiente e ao fato do instrumento para avaliação do ambiente (Craig Hospital Inventory of Environmental Factors - CHIEF) não ser específico para o contexto escolar. À luz do mesmo referencial teórico da influência de uma variável intermediária que afeta a relação entre componentes da CIF, o estudo de Rouquette et al. ${ }^{12}$ avaliou o ambiente como variável moderadora e mediadora da funcionalidade. Atividades, participação social e fatores contextuais foram mensurados por autorrelato em instrumentos padronizados em uma amostra de 548 pacientes (40 a 75 anos) com osteoartrite de joelhos não submetidos a artroplastia. Como moderador ambiental da relação entre deficiências e limitações de atividades, foram obtidas as relações familiares. Estas relações parecem atenuar deficiências do corpo em relação às atividades, $o$ que pode estar ligado a um melhor suporte social e instrumental.

Quatro estudos incluídos na revisão pesquisaram o efeito do ambiente a partir de análises qualitativas. Hammel et al. ${ }^{10}$ descreveram fatores ambientais que influenciam a participação social de 201 indivíduos com incapacidades provenientes de lesão medular, Traumatismo Crânio-Encefálico (TCE) e AVE, por meio de grupos focais. Foram identificados oito domínios de fatores ambientais: construções; naturais; tecnologias assistivas; transportes; acesso a informação e tecnologia; suporte social e atitudes; sistemas e políticas públicas; e economia. Esta influência externa pôde ser demonstrada nos níveis: imediato (família e amigos próximos), intermediário (comunidade e vizinhança) e macro (sociedade, política e economia). Ao mesmo tempo, o trabalho de Rauch et al. ${ }^{17}$ identificou os seguintes fatores ambientais: produtos e tecnologias, clima e relevo, suporte e relacionamentos, associações, sistemas e políticas publicas, e atitudes; ao avaliar 26 adultos de ambos os sexos, com lesão medular há pelo menos dois anos, de diferentes níveis e gravidades, em grupos focais e entrevistas individuais. Atitudes dos demais indivíduos de subestimação ou excessiva observação foram relatadas como barreiras. Contrastes entre os sexos foram observados em relação a suporte e relacionamentos, bem como em participar de associações, sendo algumas destas que incentivam a atividade física reportadas como demasiadamente competitivas e centradas em homens. Sveen et al. ${ }^{19}$, ao avaliarem 17 indivíduos, de 22 a 55 anos, após TCE leve dentro do contexto de um programa de reabilitação, por meio de grupos focais, observaram destacado impacto sobre a funcionalidade dos seguintes fatores ambientais: sistemas de saúde e segurança social; 
atuação de profissionais de saúde; suporte de família, amigos e comunidade; atitudes das pessoas frente ao indivíduo com deficiência; produtos e tecnologias de uso pessoal e para comunicação; e ruídos. Por fim, Randström et al. ${ }^{21}$, também utilizando método qualitativo, centralizaram suas análises nos discursos dos pacientes, consideradas as limitações, viéses inerentes e subjetividade deste tipo de pesquisa. Foi explorada a experiência de indivíduos idosos sobre os fatores ambientais que impactam em atividade e participação quando em reabilitação domiciliar. Uma pequena amostra, composta de dez idosos (3 homens e 7 mulheres), de 68 a 93 anos, com incapacidades provenientes de AVE $(\mathrm{n}=1)$ ou condições ortopédicas $(n=9)$, participou de entrevistas individuais. Para este propósito, foram identificadas 19 categorias da CIF de fatores ambientais: produtos e tecnologias (para alimentação, uso pessoal ou público, comunicação e mobilidade); clima; espaço físico em seu entorno; suporte e relacionamentos (amigos, família, comunidade, cuidadores, profissionais de saúde e animais domésticos); atitudes (de cuidadores e profissionais de saúde); políticas e serviços públicos (transporte, serviços de saúde e de suporte social).

Outros três estudos tiveram a população idosa como alvo de suas análises. Rantakokko et al. ${ }^{14}$ investigaram a associação entre life-space mobility (mobilidade em espaço cotidiano) e facilitadores e barreiras para mobilidade externa em 848 indivíduos idosos comunitários. Dos idosos, 41\% apresentaram mobilidade restrita, sendo esta associada a fatores pessoais, clínicos e ambientais. Após ajuste de idade e sexo, detectou-se papel significativo de diversos fatores ambientais para mobilidade externa (avaliado por questionário específico, previamente validado), com destaque para calçadas elevadas e presença de veículos no caminho (barreiras), bem como deslocar-se em um mesmo lado da via e o ambiente familiar (facilitadores). Ressalta-se a relevância de aspectos locais, como os climáticos ou culturais, nas respostas dadas pelos participantes e na escolha do instrumento de medida apropriado. $\mathrm{O}$ estudo desenvolvido por den Ouden et al. ${ }^{18}$ objetivou responder quais domínios da CIF contribuem para a incapacidade em Atividades de Vida Diária (AVD) básicas e instrumentais, considerando apenas o nível de urbanização, caracterizado pelo número de endereços por quilômetro quadrado, como fator ambiental. $\mathrm{O}$ modelo multivariado para explicação da incapacidade em AVD dos 537 adultos e idosos pesquisados incluiu variáveis de domínios de condições de saúde, estrutura do corpo, função do corpo e fatores pessoais; sem incluir o grau de urbanização. O componente de participação social, que poderia sofrer interferência da urbanização local, não foi avaliado pelos autores. Deve-se considerar também que indivíduos com condições de saúde variadas (doença cardiovascular, AVE, diabetes mellitus, doença pulmonar obstrutiva crônica, câncer, incontinência, fraturas e osteoartrite) foram analisados em conjunto. Os resultados reforçam a premissa de que a funcionalidade é complexa, sendo a eleição de apenas um fator do ambiente possivelmente insuficiente para a investigação de sua influência. A pesquisa de Virués-Ortega et al. ${ }^{20}$ registrou que o acesso a serviços sociais e de saúde apresenta forte e inversa associação aos níveis de incapacidade da população idosa na Espanha. Também foi relatada relação significativa entre o suporte social de um confidente e menores índices de incapacidade. Neste estudo participaram 503 idosos, com 75 anos ou mais, de nove regiões espanholas. A incapacidade foi avaliada pelo Disability Assessment Schedule (2a edição) da Organização Mundial de Saúde (WHODAS II), que enfatiza a atividade e a participação social da CIF; e as características ambientais de aspectos pontuados pelos autores de acordo com os relatos dos indivíduos, sem a utilização de um instrumento validado.

Dois outros trabalhos incluídos na revisão abordaram indivíduos com distúrbios ortopédicos. Bostan et al. ${ }^{15}$ investigaram os fatores ambientais que estão relacionados ao desempenho quando controlada a capacidade física de 296 indivíduos com distúrbios musculoesqueléticos (dor crônica difusa, lombalgia, osteoartrite, osteoporose ou artrite reumatoide). Capacidade e desempenho, assim como fatores do ambiente, foram mensurados pelas categorias e qualificadores da CIF. Para a amostra estudada, majoritariamente feminina $(80 \%)$ e com média de idade de 59 anos, foram identificados 13 fatores ambientais (físicos, sociais, políticos e de atitudes) associados significativamente ao desempenho, controlada a capacidade física. Os principais facilitadores foram: produtos e tecnologias para mobilidade e transporte interno e externos, e atuação de profissionais de saúde. Como barreiras, destacaram-se produtos ou substâncias para consumo pessoal e atitudes individuais da família imediata. Por outro lado, Gallagher et al. ${ }^{22}$ pesquisaram 148 indivíduos de 16 a 65 anos, com grandes amputações de membros, em relação às barreiras ambientais, restrições à participação e níveis de funcionalidade experimentados. O estudo, que incluiu amputados de membros superio- 
res ou inferiores, apresentou como barreiras mais relatadas as físicas, incluindo o clima, e secundariamente serviços e suporte, acesso à informação, atitudes das pessoas, transportes e a legislação vigente. Outro fator do contexto, apontado com frequência pelos indivíduos amputados como dificultador, foi a renda, apesar de controverso seu enquadramento como componente ambiental e não pessoal. Apesar do uso de instrumento validado pela avaliação da participação (WHODAS II), o domínio ambiental foi operacionalizado a partir de respostas sim ou não em itens pré-determinados. O estudo expôs apenas resultados descritivos e foi baseado em um banco de dados sobre incapacidades de população irlandesa.

Finalmente, três outros estudos na área de neurologia foram identificados. Zhang et al. ${ }^{13}$ estudaram o efeito de barreiras ambientais sobre a atividade e a participação em 639 pacientes após AVE de comunidades rurais da China $(69 \pm 11$ anos de idade), utilizando questionários validados (WHODAS II e CHIEF para mensurar incapacidades em atividades e participação e fatores ambientais, respectivamente). Após ajuste de funções neurológica e cognitiva, depressão e dados demográficos, observaram-se as barreiras físicas ou estruturais como as principais para expressão da funcionalidade. Fatores do ambiente relativos a serviços e assistência foram significativos para influenciar a participação, e não a atividade. Entretanto, deve ser considerada a dificuldade operacional de discriminação entre estes dois constructos, a atividade e a participação social, inclusive com o uso do instrumento escolhido. Neste estudo não foi detectada influência significativa de fatores políticos e de atitudes e suporte; o que pode refletir características culturais e de visão política dos chineses de áreas rurais em específico ou limitação do próprio instrumento empregado. No trabalho de Chang et al. ${ }^{16}$, que avaliou 162 adultos ( 18 a 64 anos) e 202 idosos (mais de 65 anos) de Taiwan após AVE, 65\% dos indivíduos apresentaram barreira de acesso a produtos e tecnologias para mobilidade e transporte. Ao investigarem a diferença entre pontuações de capacidade e desempenho pelo questionário WHODAS II, foi detectado efeito negativo do ambiente como um todo sobre a incapacidade global e as subescalas de mobilidade e de autocuidado. Por fim, Bouffioulx et al. ${ }^{24}$ pesquisaram 45 pacientes após AVE (29 homens e 16 mulheres, com média $69 \pm 10,7$ anos) de modo longitudinal em três momentos: agudo (1 semana após evento), pós-agudo ( 3 meses após) e crônico (6 meses após). Os fatores ambientais foram avaliados por check-list de itens da CIF, constando de quatro itens de suporte e relacionamentos e quatro de produtos e tecnologias. Resultados mostram que a assistência prestada por familiares imediatos e de profissionais de saúde foram os aspectos mais relatados no que tange ao ambiente social, assim como os dispositivos para marcha e para a realização de AVD em relação a produtos e tecnologias. Auxílios físicos e sociais foram observados em proporções similares entre as fases pós-aguda e crônica, à exceção de um declínio ao longo do tempo da assistência familiar requerida.

Limitações metodológicas foram observadas e podem ser entraves à consolidação de alguns direcionamentos descritos nesta revisão. Novas pesquisas sobre o tema com métodos bem estabelecidos, incluindo a utilização de instrumentos validados e amostras suficientemente grandes e cuidadosamente selecionadas de acordo com o propósito da investigação. Este risco de viés de seleção pode ocorrer à inclusão de pacientes com condições de saúde heterogêneas, sem o devido tratamento dos dados nas análises, ou na deficitária caracterização clínica ou funcional dos participantes. Ressalta-se a necessidade da realização de novos estudos que abordem o efeito ambiental frente à incapacidade física em áreas clínicas pouco exploradas, como na saúde cardiovascular e respiratória; uma vez que a maioria contemplou a neurologia. Também faz-se necessário o adequado emprego conceitual das denominações e componentes de funcionalidade, bem como pesquisas que avaliem a vertente positiva deste constructo e não permaneçam restritos somente às barreiras ambientais e a incapacidades geradas.

Os estudos que integraram esta revisão sistemática da literatura puderam reforçar os preceitos teóricos trazidos pela CIF que apontam para um importante papel dos fatores ambientais na expressão da funcionalidade e incapacidade humana. O ambiente físico e social, de modo geral, pôde ter sua interferência atribuída a diferentes condições clínicas, especialmente em neurologia, tanto em estudos quantitativos quanto qualitativos. Parece ser relevante que o componente ambiental seja compreendido e avaliado integralmente, pela junção dos aspectos que compõem o entorno dos indivíduos, evitando assim análises unifatoriais e isoladas. 


\section{Colaboradores}

F Athayde, EV Mancuzo e RA Corrêa participaram da concepção e elaboração do texto do artigo.

\section{Referências}

1. Palmer M, Harley D. Models and measurement in disability: an international review. Health Policy Plan 2012; 27(5):357-364.

2. Ferreira LT, Castro SS, Buchalla CM. The International Classification of Functioning, Disability and Health: progress and opportunities. Cien Saude Colet 2014; 19(2):469-474.

3. World Health Organization (WHO). International classification of functioning, disability and health: ICF. Geneva: WHO; 2001.

4. Magasi S, Wong A, Gray DB, Hammel J, Baum C, Wang CC, Heinemann, AW. Theoretical foundations for the measurement of environmental factors and their impact on participation among people with disabilities. Arch Phys Med Rehabil 2015; 96(4):569-577.

5. Francescutti C, Gongolo F, Simoncello A, Frattura L. Description of the person-environment interaction: methodological issues and empirical results of an Italian large-scale disability assessment study using an ICF-based protocol. BMC Public Health 2011; 11(Supl. 4):S11.

6. Yeung P, Towers A. An exploratory study examining the relationships between the personal, environmental and activity participation variables and quality of life among young adults with disabilities. Disabil Rehabil 2014; 36(1):63-73

7. Gasparoto M, Alpino A. Avaliação da acessibilidade domiciliar de crianças com deficiência física. Rev Bras Ed Esp 2012; 18(2):337-354.

8. Moher D, Liberati A, Tetzlaff J, Altman DG; PRISMA Group. Preferred Reporting Items for Systematic Reviews and Meta-Analyses: The PRISMA Statement. Ann Intern Med 2009; 151(4):264-269.

9. Andrade P, Ferreira F, Vasconcelos A, Lima E, Haase V. Perfil cognitivo, déficits motores e influência dos facilitadores para reabilitação de crianças com disfunções neurológicas. Rev Paul Pediatr 2011; 29(3):320-327.

10. Hammel J, Magasi S, Heinemann A, Gray DB, Stark S, Kisala P, Carlozzi NE, Tulsky D, Garcia SF, Hahn EA. Environmental barriers and supports to everyday participation: a qualitative insider perspective from people with disabilities. Arch Phys Med Rehabil 2015; 96(4):578-588.

11. Kolehmainen N, Ramsay C, McKee L, Missiuna C, Owen C, Francis J. Participation in Physical Play and Leisure in Children With Motor Impairments: Mixed-Methods Study to Generate Evidence for Developing an Intervention. Phys Ther 2015; 95(10):1374-1386.

12. Rouquette A, Badley EM, Falissard B, Dub T, Leplege A, Coste J. Moderators, mediators, and bidirectional relationships in the International Classification of Functioning, Disability and Health (ICF) framework: An empirical investigation using a longitudinal design and Structural Equation Modeling (SEM). Soc Sci Med 2015; 135:133-142.

13. Zhang L, Yan T, You L, Li K. Barriers to activity and participation for stroke survivors in rural China. Arch Phys Med Rehabil 2015; 96(7):1222-1228.

14. Rantakokko M, Iwarsson S, Portegijs E, Viljanen A, Rantanen T. Associations between environmental characteristics and life-space mobility in community-dwelling older people. J Aging Health 2015; 27(4):606-621. 
15. Bostan C, Oberhauser C, Stucki G, Bickenbach J, Cieza A. Which environmental factors are associated with performance when controlling for capacity? J Rehabil Med 2014; 46(8):806-813.

16. Chang KH, Lin YN, Liao HF, Yen CF, Escorpizo R, Yen TH, Liou TH. Environmental effects on WHODAS 2.0 among patients with stroke with a focus on ICF category e120. Qual Life Res 2014; 23(6):1823-1831.

17. Rauch A, Fekete C, Cieza A, Geyh S, Meyer T. Participation in physical activity in persons with spinal cord injury: a comprehensive perspective and insights into gender differences. Disabil Health J 2013; 6(3):165-176.

18. den Ouden M, Schuurmans M, Mueller-Schotte S, Brand J, van der Schouw Y. Domains 18. Contributing to Disability in Activities of Daily Living. J Am Med Dir Assoc 2013; 14(1):18-24.

19. Sveen U, Ostensjo S, Laxe S, Soberg H. Problems in functioning after a mild traumatic brain injury within the ICF framework: the patient perspective using focus groups. Disabil Rehabil 2013; 35(9):749-757.

20. Virues-Ortega J, de Pedro-Cuesta J, del Barrio JL, Almazan-Isla J, Bergareche A, Bermejo-Pareja F, Fernández-Mayoralas G, García FJ, Garre-Olmo J, Gascon-Bayarri J, Mahillo I, Martínez-Martín P, Mateos R, Rodríguez F, Rojo-Pérez F, Avellanal F, Saz P, Seijo-Martínez M, Spanish Epidemiological Study Group on Aging.. Medical, environmental and personal factors of disability in the elderly in Spain: a screening survey based on the International Classification of Functioning. Gac Sanit 2011; 25 (Supl. 2):29-38.

21. Randström K, Asplund K, Svedlund M. Impact of environmental factors in home rehabilitation - a qualitative study from the perspective of older persons using the International Classification of Functioning, Disability and Health to describe facilitators and barriers. Disabil Rehabil 2012; 34(9):779-787.

22. Gallagher P, O’Donovan MA, Doyle A, Desmond D. Environmental barriers, activity limitations and participation restrictions experienced by people with major limb amputation. Prosthet Orthot Int 2011; 35(3):278284.

23. Rosenberg L, Ratzon NZ, Jarus T, Bart O. Perceived environmental restrictions for the participation of children with mild developmental disabilities. Child Care Health Dev 2012; 38(6):836-843.

24. Bouffioulx E, Arnould C, Thonnard JL. Satisfaction with activity and participation and its relationships with body functions, activities, or environmental factors in stroke patients. Arch Phys Med Rehabil 2011; 92(9):1404-1410.

25. Furtado SR, Sampaio RF, Kirkwood RN, Vaz DV, Mancini MC. Moderating effect of the environment in the relationship between mobility and school participation in children and adolescents with cerebral palsy. Braz J Phys Ther 2015; 19(4):311-319.

Artigo apresentado em 24/08/2016

Aprovado em 12/04/2017

Versão final apresentada em 14/04/2017 